\title{
Nonlinear Optical Processes in Organic and Polymer Structures
}

\author{
A. F. GARITO and K. Y. WONG \\ Department of Physics, University of Pennsylvania, \\ Philadelphia, PA 19104-6396, U.S.A.
}

(Received August 29, 1986)

\begin{abstract}
Large, ultrafast second and third order nonlinear optical properties have been demonstrated in a large number of organic and polymer structures. Such advances are important to the fields of nonlinear optics and optical device technologies. The organic and polymer systems can be formed as organizable designed structures possessing a number of special primary properties that include wideband electronic polarizations whose microscopic origin resides in highly charge correlated virtual exçitations. These ultrafast excitations occur on individual molecular, or polymer chain, sites whose condensed assemblies are described by their orientational distribution functions. In this regard, the results of two recent studies are reviewed.

KEY WORDS Nonlinear Optical Process / Orientational Distribution

Functions / Third Harmonic Generation /
\end{abstract}

Organic and polymer structures exhibit unusually large, ultrafast second and third order nonlinear optical properties that are important to the fields of nonlinear optics and optical device technologies. These important properties have been demonstrated in a large number of structures, phases and states that include, as previously reviewed, ${ }^{1-9}$ organic crystals and films, conjugated polymers, monomolecular films, liquid crystals, liquid crystal polymers, and, more recently, high performance ordered polymers ${ }^{10,11}$ and polymer glasses. ${ }^{12}$ Accordingly, these developments together with demonstrations of phase conjugate wave generation, optical bistability, and associative memory networks have stimulated considerable growth in research and development activities. A large variety of potential applications in advanced optical technologies are being actively pursued that involve optical signal processing and computing, image reconstruction, data storage, and telecommunications.

In recent years, theoretical and experimental interest has centered on the origin of the exceptional second ${ }^{13}$ and third order ${ }^{14}$ nonlinear optical responses observed for certain organic and polymeric structures. In each case, the origin of these nonlinear responses resides in the highly charge correlated virtual excitation of the conjugated $\pi$-electron structure. The $\pi$-electron states are true many-body states because electron correlations due to natural repulsive Coulomb interactions tend to localize the otherwise delocalized electrons. Electron correlations play an important role in the nonlinear optical responses of conjugated organic structures, ${ }^{1-3,13,15,16}$ and their description of the nonlinear optical susceptibilities differs markedly from independent particle models. In calculating the sign, magnitude and dispersion of microscopic higher order susceptibilities, suitable descriptions of the virtual excitations to the correlated $\pi$-electron excited states can be obtained by self consistent field molecular orbital procedures (SCF-MO) that include single and double configuration interactions (SDCI) to account for electron correlations. ${ }^{1-3,13,15,16}$ 
The macroscopic nonlinear optical susceptibilities of a medium is related to their corresponding microscopic susceptibilities through orientational statistical averaging. Thus, the understanding of the nonlinear properties of the macroscopic medium is reduced to experimental and theoretical studies of the orientational distribution function of the molecular, or polymeric, units making up the nonlinear medium and their corresponding microscopic nonlinear susceptibilities. In Section I of this article, we briefly summarize several nonlinear optical processes. Section II contains a discussion of the orientational distribution function and third harmonic generation applicable to uniaxial systems such as nematic liquid crystal phases and uniaxially oriented polymers. Section III illustrates the electronic origin of the second order susceptibility of the fundamentally important case of conjugated linear chain structures.

\section{NONLINEAR OPTICAL PROCESSES}

Nonlinear optical responses are expressed through the constitutive relation for the dielectric polarization $P(t)$ of a nonlinear medium in an intenșe optical field $E(t)=\operatorname{Re}\left(E e^{i \omega t}\right)^{17}$

$$
P(t)=\chi^{(1)} E(t)+\chi^{(2)} E^{2}(t)+\chi^{(3)} E^{3}(t)+\cdots
$$

The first and third order terms in odd powers of $E(t)$ are common for all materials. $\chi^{(1)}$ represents linear optics, and $\chi^{(3)}$ third order nonlinear processes. Important examples include third harmonic generation $(\omega+\omega+\omega=$ $3 \omega)$ and the optical Kerr effect $(\omega-\omega+\omega=$ $\omega)$. The second order response $\chi^{(2)}$ occurs only in noncentrosymmetric media; that is, those lacking a natural center of inversion symmetry. In general for second order processes, fundamental frequencies at $\omega_{1}$ and $\omega_{2}$ combine to create a third frequency at $\omega_{3}\left(\omega_{1} \pm \omega_{2}=\right.$ $\left.\omega_{3}\right)$. For example, having $\omega_{1}$ and $\omega_{2}$ the same, the sum leads to second harmonic generation $(\omega+\omega=2 \omega)$, and the difference, optical rectification $(\omega-\omega=0)$. Moving one frequency down to $\mathrm{d} c$ results in the linear electrooptic effect $(\omega+0=\omega)$. Thus, there are a large number of important nonlinear optical processes over the entire frequency range, and the frequency dependences, or dispersions, of $\chi^{(2)}$ and $\chi^{(3)}$ are fundamentally important properties of the nonlinear medium.

For large classes of conjugated molecules and polymer structures, the nonlinear optical responses are dominated by lossless virtual excitations of the $\pi$-electron states, especially those possessing large charge correlations. Currently, the $\pi$-electron excitations are viewed as occurring on individual molecular, or polymer, sites and providing macroscopic sources of nonlinear response through the corresponding on-site microscopic nonlinear optical susceptibility. Since intramolecular bonding interactions $\left(100 \mathrm{kcal} \mathrm{mol}^{-1}\right)$ are much stronger than relatively weak van der Waals intermolecular interactions $(1 \mathrm{kcal}$ $\mathrm{mol}^{-1}$ ), each molecular, or polymer, unit is essentially an independent source of nonlinear response. Each term in the macroscopic polarization of eq 1 can thus be expressed as comprised of the corresponding microscopic nonlinear optical response

$$
p(t)=\alpha E(t)+\beta E^{2}(t)+\gamma E^{3}(t)+\cdots
$$

where $\alpha$ is the linear polarizability, and $\beta$ and $\gamma$ are the second and third order nonlinear electronic susceptibilities, respectively. The second and third order macroscopic nonlinear susceptibilities $\chi^{(2)}\left(-\omega_{3} ; \omega_{1}, \omega_{2}\right)$ and $\chi^{(3)}\left(-\omega_{4} ; \omega_{1}\right.$, $\left.\omega_{2}, \omega_{3}\right)$ can be related to the corresponding microscopic susceptibilities $\beta\left(-\omega_{3} ; \omega_{1}, \omega_{2}\right)$ and $\gamma\left(-\omega_{4} ; \omega_{1}, \omega_{2}, \omega_{3}\right)$ by considering an ensemble average over the orientational distribution and local field correction factors

$$
\begin{array}{rl}
\chi_{i j k}^{(2)}=N & \left\langle R_{i m^{\prime}} R_{j n^{\prime}} R_{k o^{\prime}} f_{m^{\prime} i^{\prime}}^{\omega_{3}} \beta_{i^{\prime} j^{\prime} k^{\prime}} f_{j^{\prime} n^{\prime}}^{\omega_{1}} f_{k^{\prime} o^{\prime}}^{\omega_{2}}\right\rangle \\
\chi_{i j k l}^{(3)}=N & N\left\langle R_{i m^{\prime}} R_{j n^{\prime}}, R_{k o^{\prime}} R_{l p^{\prime}} f_{m^{\prime} i^{\prime}}^{\omega_{4}} \gamma_{i^{\prime} j^{\prime} k^{\prime} l^{\prime}}\right. \\
& \left.\times f_{j^{\prime} n^{\prime}}^{\omega_{1}} f_{k^{\prime} o^{\prime}}^{\omega_{2}} f_{l^{\prime} p^{\prime}}^{\omega_{3}}\right\rangle
\end{array}
$$


where the unprimed and primed indices correspond to the laboratory and the molecule fixed frame, respectively; $N$, the number of sites per unit volume; $R$, the rotation matrix relating the laboratory and the molecular frame; $f^{\omega_{i}}$ the local field correction factor at frequency, $\omega_{i}$; and the bracket $\langle>$ represents an ensemble average over the orientational distribution. In the rigid lattice gas approximation, the orientation distribution can be described by an orientational distribution function $F(\phi, \theta, \psi)$ which is the probability for a molecular or polymer unit having an orientation with Euler angles $(\phi, \theta, \psi)$ with respect to the laboratory frame. The orientational ensemble average for a general tensor $T$ can then be expressed as

$$
\begin{array}{r}
\left\langle T_{\mathrm{abc}} \ldots\right\rangle=\int_{0}^{2 \pi} \mathrm{d} \phi \int_{0}^{\pi} \sin \theta \mathrm{d} \theta \int_{0}^{2 \pi} \mathrm{d} \psi \\
T_{\mathrm{abc}} \ldots F(\phi, \theta, \psi)
\end{array}
$$

Thus the understanding of the origin of the macroscopic $\chi^{(2)}$ or $\chi^{(3)}$ is reduced to experimental and theoretical studies of the corresponding microscopic $\beta$, or $\gamma$, of single molecular, or polymer chain, units making up the nonlinear organic medium and their related orientational distribution functions $F(\phi, \theta, \psi)$.

In the next section of this paper, we discuss the study of the orientational distribution function, $F(\phi, \theta, \psi)$ and third harmonic generation, $\chi_{i j k l}^{(3)}(-3 \omega ; \omega, \omega, \omega)$, and, in Section III, we will describe a recently completed study of the microscopic susceptibility $\chi_{i j k}(-2 \omega ; \omega, \omega)$ of conjugated linear chain structures.

\section{ORIENTATIONAL DISTRIBUTION FUNCTIONS AND THIRD HAR- MONIC GENERATION}

The orientational distribution function assumes a different functional form depending on the symmetry of the material medium. For organic or polymer single crystals, the molecular, or polymer, units are essentially fixed in the lattice with fixed orientation, apart from negligible thermal fluctuations. The orientational distribution function in this case is the normalized sum of delta functions at the orientations allowed by the corresponding crystal symmetry group. Equations 3 and 4 can then be expressed as

$$
\begin{aligned}
\chi_{i j k}^{(2)}=N_{u} \sum_{s=1}^{P} R_{i m^{\prime}}^{s}, R_{j n^{\prime}}^{s} R_{k o^{\prime}}^{s} f_{m^{\prime} i^{\prime}}^{\omega_{3}} \beta_{i^{\prime} j^{\prime} k^{\prime}}^{s} f_{j^{\prime} n^{\prime}}^{\omega_{1}} f_{k^{\prime} o^{\prime}}^{\omega_{2}} \\
\chi_{i j k l}^{(3)}=N_{u} \sum_{s=1}^{P} R_{i m^{\prime}}^{s} R_{j n^{\prime}}^{s} R_{k o^{\prime}}^{s}, R_{l p^{\prime}}^{s} \\
\quad \times f_{m^{\prime} i^{\prime}}^{\omega_{4}} \gamma_{i^{\prime} j^{\prime} k^{\prime} l^{\prime} l^{\prime}} f_{j^{\prime} n^{\prime}}^{\omega_{1}} f_{k^{\prime} o^{\prime}}^{\omega_{2}} f_{l^{\prime} p^{\prime}}^{\omega_{3}}
\end{aligned}
$$

where $N_{u}$ is the number of unit cells per unit volume, and the summation is over all $p$ molecules in a unit cell. Each molecule indexed by $s$ in the unit cell usually has a different orientation, and thus $R_{i a}^{s}$ is the rotation matrix from the body axis of the $s$ molecule in the unit cell to the laboratory axis. $\beta_{i^{\prime} j^{\prime} k^{\prime}}^{s}$ and $\gamma_{i^{\prime} j^{\prime} k^{\prime} l^{\prime}}^{s}$ are the respective microscopic susceptibility tensor components corresponding to the body axis of the $s$ molecule. The macroscopic nonlinear optical susceptibility of an organic or polymer single crystal is thus expressed in terms of the corresponding microscopic susceptibility and the crystal symmetry group.

For a liquid, or isotropic polymer, there is no preferred orientational direction for molecular or polymer units in the media, and all directions are equally probable. The orientational distribution function is equal to a simple constant $1 / 8 \pi^{2}$ by normalization. The second order macroscopic susceptibility $\chi^{(2)}$ vanishes in this case due to inversion symmetry. The third order macroscopic susceptibility $\chi^{(3)}$ can be evaluated through eq 4 to obtain

$$
\begin{aligned}
\chi_{z z z z}^{(3)}= & \frac{1}{5} N f^{\omega_{1}} f^{\omega_{2}} f^{\omega_{3}} f^{\omega_{4}}\left(\gamma_{x^{\prime} x^{\prime} x^{\prime} x^{\prime}}+\gamma_{y^{\prime} y^{\prime} y^{\prime} y^{\prime}}\right. \\
& +\gamma_{z^{\prime} z^{\prime} z^{\prime} z^{\prime}}+\gamma_{x^{\prime} x^{\prime} y^{\prime} y^{\prime}}+\gamma_{y^{\prime} x^{\prime} x^{\prime} y^{\prime}}+\gamma_{y^{\prime} y^{\prime} z^{\prime} z^{\prime}} \\
& \left.+\gamma_{z^{\prime} y^{\prime} y^{\prime} z^{\prime}}+\gamma_{z^{\prime} z^{\prime} x^{\prime} x^{\prime}}+\gamma_{x^{\prime} z^{\prime} z^{\prime} x^{\prime}}\right)
\end{aligned}
$$


where the laboratory $z$-axis is now degenerate in all directions for the isotropic case.

The crystal state and the liquid state represent the two natural limits where the molecular, or polymer, units are either fixed, or completely random in orientation. In general, for partially ordered phases intermediate between these two limiting cases, the orientational distribution function assumes a more complex form. For example, for the equivalent cases of a nematic liquid crystal, nematic liquid crystal polymer, or uniaxially oriented polymer, unlike a crystal, the system has an arbitrary translational symmetry; and, unlike a liquid, the system does not have arbitrary rotational symmetry. Instead the molecular, or polymer chain, units are oriented in a distribution centered in a preferred direction. We will refer to this direction as the symmetry axis.

The orientational distribution function can be simplified by imposing the symmetry of the macroscopic phase. For a uniaxial phase with its symmetry axis parallel to the $z$-axis, the orientational distribution function $F(\phi, \theta, \psi)$ is independent of $\phi$. If each molecular, or polymer, unit is cylindrically symmetric along its long molecular, or polymer chain, axis, the orientational distribution function is independent of $\psi$ and can thus be described by a single variable function $F(\theta) . F(\theta)$ can then be expanded in terms of Legendre polynomials $P_{l}(\cos \theta)$

$$
F(\theta)=\sum_{l=0}^{\infty} \frac{2 l+1}{2} A_{l} P_{l}(\cos \theta)
$$

with

$$
A_{l}=\int_{0}^{\pi} \sin \theta \mathrm{d} \theta F(\theta) P_{l}(\cos \theta) \equiv\left\langle P_{l}\right\rangle
$$

where $\left\langle P_{l}\right\rangle$ is defined as the $l$ th order parameter. In the absence of piezoelectricity for this phase, then only $\left\langle P_{l}\right\rangle$ with $l$ even are nonzero. Since $\left\langle P_{0}\right\rangle$ is related only to the normalization of the distribution function, the lowest order nonvanishing order parameters are thus $\left\langle P_{2}\right\rangle$ and $\left\langle P_{4}\right\rangle$.

Understanding of the orientational distribution in a nematic liquid crystal, nematic liquid crystal polymer, or uniaxially oriented polymer is thus reduced to the determination of the order parameters $\left\langle P_{l}\right\rangle$. In general, the orientational average of an $l$ th rank tensor involves up to the $l$ th order of the statistical average of the Legendre polynomial $\left\langle P_{l}\right\rangle$. The lowest order parameters $\left\langle P_{2}\right\rangle$ and $\left\langle P_{4}\right\rangle$ are thus involved in third order nonlinear optical processes. To illustrate this case, we will discuss an experimental determination of the order parameter $\left\langle P_{2}\right\rangle$ and $\left\langle P_{4}\right\rangle$ for the welldefined nematic liquid crystal case using optical third harmonic generation. ${ }^{18}$

Measurements of the third harmonic susceptibility are performed on those components along the principal axis of the bulk phase. The dependence of the macroscopic tensors $\chi_{z z z z}^{(3)}$ and $\chi_{x x x x}^{(3)}$ on the microscopic tensor $\gamma_{i^{\prime} j^{\prime} k^{\prime} l^{\prime}}$, where $z$ is along the unique axis, can be obtained by evaluating eq 4 . The results are ${ }^{18}$

$$
\begin{aligned}
& \chi_{z z z z}^{(3)}=N\left(\delta+2 \xi\left\langle P_{2}\right\rangle+8 \eta\left\langle P_{4}\right\rangle\right) \\
& \chi_{x x x x x}^{(3)}=N\left(\delta-\xi\left\langle P_{2}\right\rangle+3 \eta\left\langle P_{4}\right\rangle\right) \\
& \chi_{\text {iso }}^{(3)}=N \delta
\end{aligned}
$$

where

$$
\begin{aligned}
\delta= & \frac{1}{5}\left(\gamma_{z^{\prime} z^{\prime} z^{\prime} z^{\prime}}+\gamma_{x^{\prime} x^{\prime} x^{\prime} x^{\prime}}+\gamma_{y^{\prime} y^{\prime} y^{\prime} y^{\prime}}+\gamma_{z^{\prime} z^{\prime} x^{\prime} x^{\prime}}\right. \\
& +\gamma_{x^{\prime} z^{\prime} z^{\prime} x^{\prime}}+\gamma_{z^{\prime} z^{\prime} y^{\prime} y^{\prime}}+\gamma_{y^{\prime} z^{\prime} z^{\prime} y^{\prime}}+\gamma_{x^{\prime} x^{\prime} y^{\prime} y^{\prime}} \\
& \left.+\gamma_{y^{\prime} x^{\prime} x^{\prime} y^{\prime}}\right) \\
\xi= & \frac{1}{14}\left(4 \gamma_{z^{\prime} z^{\prime} z^{\prime} z^{\prime}}-2 \gamma_{x^{\prime} x^{\prime} x^{\prime} x^{\prime}}-2 \gamma_{y^{\prime} y^{\prime} y^{\prime} y^{\prime}}+\gamma_{z^{\prime} z^{\prime} x^{\prime} x^{\prime}}\right. \\
& +\gamma_{x^{\prime} z^{\prime} z^{\prime} x^{\prime}}+\gamma_{z^{\prime} z^{\prime} y^{\prime} y^{\prime}}+\gamma_{y^{\prime} z^{\prime} z^{\prime} y^{\prime}}-2 \gamma_{x^{\prime} x^{\prime} y^{\prime} y^{\prime}} \\
& \left.-2 \gamma_{y^{\prime} x^{\prime} x^{\prime} y^{\prime}}\right) \\
\eta= & \frac{1}{280}\left(8 \gamma_{z^{\prime} z^{\prime} z^{\prime} z^{\prime}}+3 \gamma_{x^{\prime} x^{\prime} x^{\prime} x^{\prime}}+3 \gamma_{y^{\prime} y^{\prime} y^{\prime} y^{\prime}}\right. \\
& -12 \gamma_{z^{\prime} z^{\prime} x^{\prime} x^{\prime}}-12 \gamma_{x^{\prime} z^{\prime} z^{\prime} x^{\prime}}-12 \gamma_{z^{\prime} z^{\prime} y^{\prime} y^{\prime}} \\
& -12 \gamma_{y^{\prime} z^{\prime} z^{\prime} y^{\prime}}+3 \gamma_{x^{\prime} x^{\prime} y^{\prime} y^{\prime}}+3 \gamma_{y^{\prime} x^{\prime} x^{\prime} y^{\prime}}
\end{aligned}
$$

$\chi_{\text {iso }}^{(3)}$ is the susceptibility for the isotropic phase 


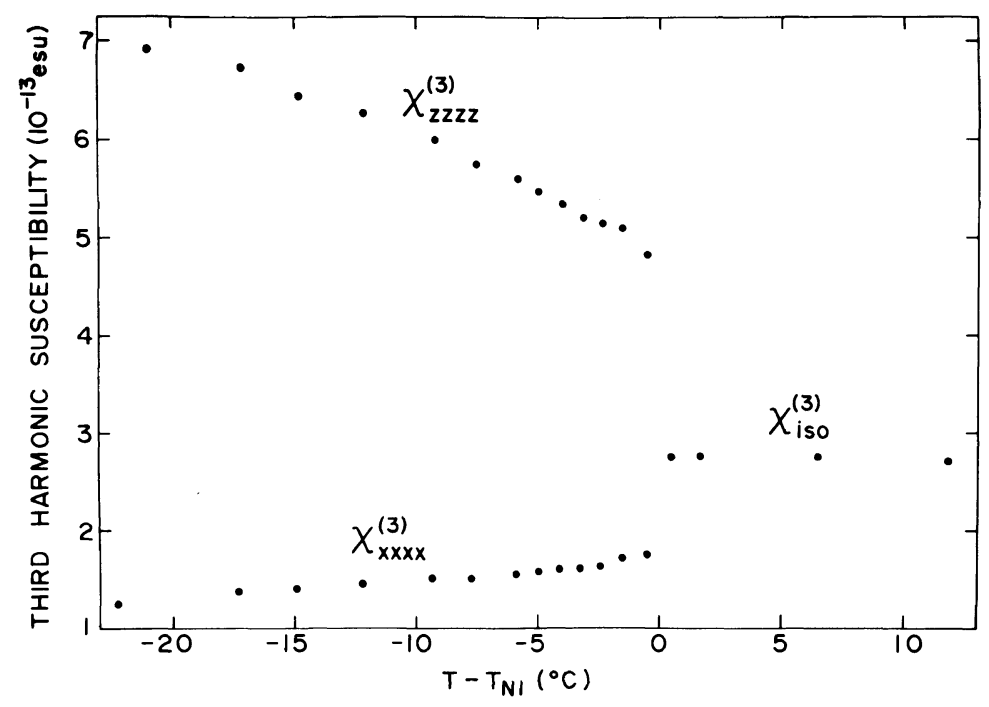

Figure 1. Measured third harmonic susceptibility of MBBA liquid crystal as function of temperature; $z$ axis and $x$-axis are parallel and perpendicular to the director, respectively (ref 18).

where $\left\langle P_{2}\right\rangle$ and $\left\langle P_{4}\right\rangle$ vanish. We assume that the change in density as a function of temperature and through the phase transition is negligible. We see from eq 9 that the third order susceptibility $\chi_{z z z z}^{(3)}$ and $\chi_{x x x x}^{(3)}$ are functions of temperature as a result of the temperature dependence of $\left\langle P_{2}\right\rangle$ and $\left\langle P_{4}\right\rangle$ in the nematic phase. They become temperature independent after a phase transition to the isotropic phase. Measurement of $\chi_{z z z z}^{(3)}, \chi_{x x x x}^{(3)}$, and $\chi_{\text {iso }}^{(3)}$ allows simultaneous determination of the order parameter $\left\langle P_{2}\right\rangle$ and $\left\langle P_{4}\right\rangle$.

The experimental design for the measurement of the third harmonic susceptibility is described in ref 18 . The homogeneously aligned liquid crystal sample was contained between two surface treated glass plates spaced by a glass fiber, forming a wedged shape. The liquid crystal $N$-( $p$-methoxybenzylidene)- $p$ butylaniline (MBBA) was chosen as the material for investigation so that direct comparison could be made with earlier experimental results, especially Raman scattering data. ${ }^{19}$ The sample was mounted on a temperature stabilized heating cell which was in turn mounted inside a vacuum chamber. The

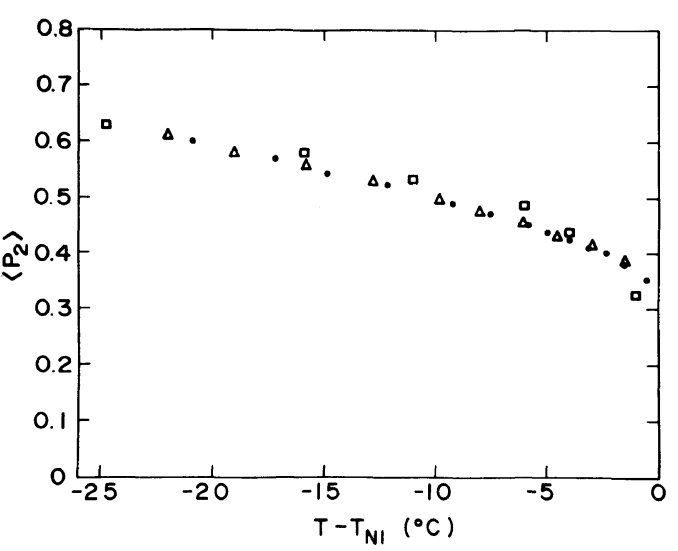

Figure 2. $\left\langle P_{2}\right\rangle$ of MBBA as a function of temperature. 0 , from third harmonic generation measurements (ref 18); $\square$, from NMR measurement (ref 20); $\triangle$, from diamagnetic anisotropy measurement (ref 21).

wedged Maker fringe method was employed for the relative determination of the $\chi^{(3)}$ of the liquid crystal with respect to the reported value of BK-7 glass.

The results of the measurement of the third harmonic susceptibility $\chi^{(3)}$ for the MBBA is given in Figure 1. The order parameter $\left\langle P_{2}\right\rangle$ and $\left\langle P_{4}\right\rangle$ are calculated from the experimental results of the third harmonic susceptibility. ${ }^{18}$ 


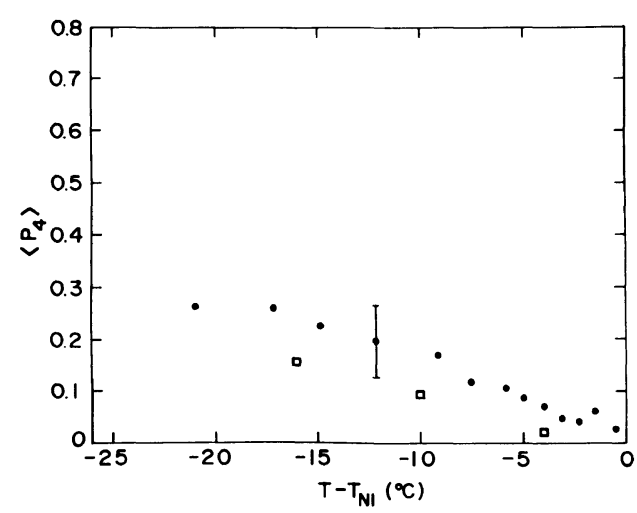

Figure 3. $\left\langle P_{4}\right\rangle$ of MBBA as function of temperature. , from third harmonic generation measurements (ref 18); $\square$, from Raman scattering measurements (ref 19).

The results are shown in Figures 2 and 3. Also shown in the figures are results from earlier reported studies using $\mathrm{NMR},{ }^{20}$ diamagnetic anisotropy ${ }^{21}$ and Raman scattering methods. ${ }^{19}$ Our results agree very well with previously reported values. Thus, through third harmonic generation measurements of the well-defined MBBA system, we can conclude from their example that the order parameters $\left\langle P_{2}\right\rangle$ and $\left\langle P_{4}\right\rangle$ of the general uniaxial case of nematic liquid crystal, nematic liquid crystal polymer, and uniaxially oriented polymer can be determined by optical third harmonic studies.

\section{ELECTRONIC ORIGIN OF THE MICROSCOPIC SECOND ORDER OPTICAL SUSCEPTIBILITY}

In this section, we address the fundamental problem of understanding the origin of large, nonresonant second order nonlinear optical susceptibilities of conjugated $\pi$-electron organic and polymer structures. As previously described, in noncentrosymmetric resonant ring structures, such as 2-methyl-4-nitroaniline (MNA), large charge asymmetries occur in the virtual excitations of $\pi$-electronic states due to electron correlations which thus determine the magnitude, sign and dispersion of the microscopic second order nonlinear optical response $\beta_{i j k}\left(-\omega_{3} ; \omega_{1}, \omega_{2}\right){ }^{1-3,13,15,22}$ Important to third order processes in centrosymmetric structures such as conjugated linear chains, the correlated motion of $\pi$-electrons leads to formation of two photon excited states, ${ }^{24}$ for example, in centrosymmetric trans linear polyenes $^{25}$ (alternating single and double bonds) and polyenynes (alternating single, double and triple bonds). ${ }^{3,7,26}$

Linear polyenes and polyenynes represent the finite chain limit to the corresponding quasi-one dimensional conjugated polymer chain, and phase matched second harmonic generation, for example, has been demonstrated for noncentrosymmetric conjugated polymer structures. ${ }^{1-3,7,27}$ In this regard, for the fundamentally important case of a noncentrosymmetric conjugated linear chain, NMDVDA [trans-trans 1-(4-methylphenyl)-8(4'-nitrophenyl)-1,7-diene-3,5-octadiyne $]^{3}$, the first results have been obtained ${ }^{16}$ for the fre-

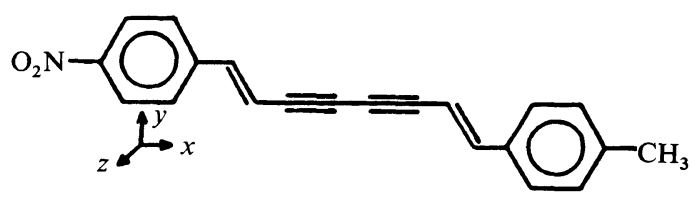

quency dependent $\beta_{i j k}(-2 \omega ; \omega, \omega)$ as determined by DC induced second harmonic generation (DCSHG) measurements and corresponding all valence electron, self consistent field molecular orbital calculations (SCF-MO) with single and double configuration interactions (SDCI) to account for electron correlations.

The vector part $\beta_{x}(-2 \omega ; \omega, \omega)$ of $\beta_{i j k}(-2 \omega ; \omega, \omega)$ was experimentally determined by $\mathrm{DCSHG}^{15,23,28,29}$ liquid solution measurements of NMDVDA dissolved in dioxane using wedge cell and infinite dilution methods described earlier. ${ }^{15,29}$ The measured macroscope susceptibility $\Gamma_{\mathrm{L}}$ for a two component solution of solvent (0) and solute (1) given by the infinite dilution equation ${ }^{29}$ 


$$
\begin{gathered}
\frac{\left(2 \varepsilon_{0}+n_{1}^{2}\right)\left(2 n_{0}^{2}+n_{1}^{2}\right)^{3} M_{1}}{\left(n_{1}^{2}+2\right)^{4} n_{0}^{6} \varepsilon_{0}}\left\{\left.v_{0} \frac{\partial \Gamma_{\mathrm{L}}}{\partial w}\right|_{0}+\left.\Gamma_{0} \frac{\partial v}{\partial w}\right|_{0}\right. \\
+v_{0} \Gamma_{0}-v_{0} \Gamma_{0}\left[\left.\frac{1}{n_{0}^{2}} \frac{\partial n^{2}}{\partial w}\right|_{0}\right. \\
\left.\left.+\left.\left(\frac{1}{\varepsilon_{0}}-\frac{2}{2 \varepsilon_{0}+n_{0}^{2}}\right) \frac{\partial \varepsilon}{\partial w}\right|_{0}\right]\right\}=N_{\mathrm{A}} \gamma_{1}^{\prime}
\end{gathered}
$$

where $\gamma_{i}$ is $\beta_{i} \mu_{i} / 5 k_{\mathrm{B}} T$ with $\mu_{i}$ the dipole moment; $w$ solute weight fraction; $v$ is the specific volume; $\varepsilon$, static dielectric constant; $n$, index of refraction; and $N_{\mathrm{A}}$, Avogadro's number. Solvent induced shifts in solute excitation energies for $\beta_{x}$ were analyzed as described previously. ${ }^{15}$ The dipole moment $(5.1 \pm 0.3 \mathrm{D})$ of NMDVDA in dioxane was standardly determined by infinite dilution procedures. ${ }^{29}$

The experimental values of $\beta_{x}$ for NMDVDA as a function of frequency are listed in Table I and plotted in Figure 4. With increased frequency, the positive $\beta_{x}$ values starting at approximately $40 \times 10^{-30}$ esu increase smoothly to greater than $300 \times$ $10^{-30}$ esu in approaching the $2 \omega$ singularity which corresponds to the observed excitation energy $3.31 \mathrm{eV}(0.375 \mu \mathrm{m})$ of the major absorption peak of NMDVDA in dioxane (Figure 1 insert).

The frequency dependent $\beta_{i j k}$ components and singlet-singlet excitation spectrum of NMDVDA were theoretically calculated by the original SCF-MO-CI method ${ }^{3,13}$ in the rigid lattice $\mathrm{CNDO} / s$ approximation. Included were all (16) possible singly excited (SCI) and 100 lowest energy doubly excited configurations (DCI) involving the four highest occupied $\pi$-orbitals and the four lowest virtual $\pi$ orbitals along with the SCF ground state. The results were unchanged by increased $\mathrm{CI} .{ }^{16}$

For $\quad \beta_{x}=\beta_{x x x}+1 / 3\left(\beta_{x y y}+\beta_{x z z}+2 \beta_{z z x}+\right.$ $\left.2 \beta_{y x x}\right)$ ), the theoretical gas phase components are, for example, at $0.650 \mathrm{eV}(\lambda=1.907 \mu \mathrm{m})$ $\beta_{x x x}: 34.2 ; \beta_{x y y}: 1.9 ; \beta_{x z z}: 0 ; \beta_{z z x}: 0$; and $\beta_{y x x}: 1.4$ $\left(\times 10^{-30} \mathrm{esu}\right) .{ }^{30}$ The calculated dispersion $\mathrm{cu}-$ rve for $\beta_{x}$ is plotted as the solid line in Figure 4
Table I. Frequency-dependent experimental values $\beta_{x}^{\exp }$ of NMDVDA dissolved in 1,4-dioxane

\begin{tabular}{cccc}
\hline$\lambda$ & $\hbar \omega$ & $\left.\frac{\partial \Gamma_{\mathbf{L}}}{\partial w}\right|_{0}$ & \multicolumn{1}{c}{$\frac{\beta_{x}^{\exp }}{10^{-30} \mathrm{esu}}$} \\
\hline$\mu \mathrm{m}$ & $\overline{\mathrm{eV}}$ & $\frac{10^{-12} \mathrm{esu}}{}$ & \\
\hline 1.907 & 0.650 & $6.7 \pm 0.3$ & $38.8 \pm 1.7$ \\
1.064 & 1.165 & $16.1 \pm 0.8$ & $89.0 \pm 4.5$ \\
0.909 & 1.364 & $35.2 \pm 1.7$ & $195.2 \pm 9.7$ \\
0.880 & 1.409 & $43.5 \pm 2.0$ & $241.6 \pm 11.2$ \\
0.860 & 1.442 & $51.8 \pm 2.8$ & $287.6 \pm 15.5$ \\
\hline
\end{tabular}

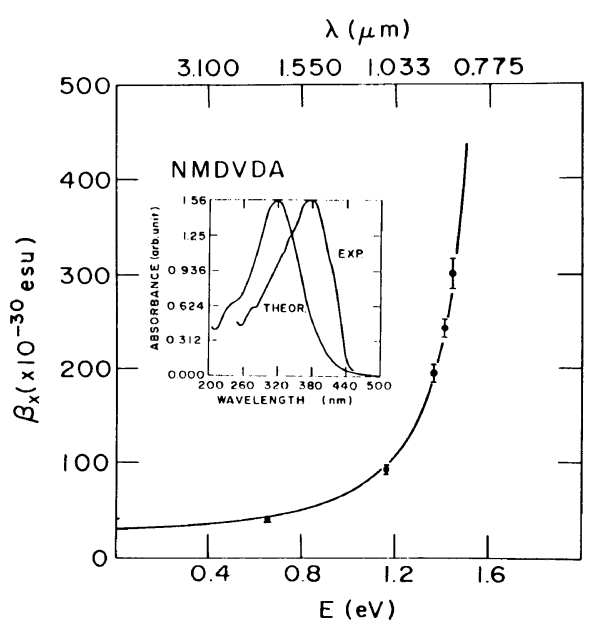

Figure 4. Frequency dependent $\beta_{x}(-2 \omega ; \omega, \omega)$ for NMDVDA: ( $\bar{\phi})$ experiment; (-) theory. Comparison of singlet-singlet excitations spectra with large oscillator strength transitions to states I, III, and IV. Dielectric shift due to dipole mediated solvent interactions (ref 16).

after accounting for dipole mediated solvent induced shifts. ${ }^{15}$ The comparison between experiment and theory for the magnitude, sign, and dispersion of $\beta_{x}$ is quite satisfactory.

The largest $\beta_{x}$ component is $\beta_{x x x}$, having diagonal $\beta_{x x x}^{n n}$ and off-diagonal $\beta_{x x x}^{n n^{\prime}}$ contributions of $54 \%$ and $46 \%$, respectively. The diagonal component of $\beta_{x x x}^{n n}$ is given by the expression

$$
\begin{aligned}
\beta_{x x x}^{n n}= & -\frac{3}{2 \hbar^{2}}\left\{\sum_{n} \mu_{n g}^{x} \mu_{n g}^{x} \Delta \mu_{n}^{x} \omega_{n g}^{2}\left(\omega_{n g}^{2}-\omega^{2}\right)^{-1}\right. \\
& \left.\times\left(\omega_{n g}^{2}-4 \omega^{2}\right)^{-1}\right\}
\end{aligned}
$$


where $\mu_{n g}^{x}=-\mathrm{e} r_{n g}^{x}$ is the $x$-component of the transition moment between the ground $(g)$ and excited (n) states; $\Delta \mu_{n g}^{x}=-\mathrm{e} \Delta r_{n g}^{x}$ is the $x$ component of the dipole moment difference between the ground and excited states, and the sum is over all states. The dominant NMDVDA singlet excited states are I, III and IV with calculated gas phase values, respectively, for $\omega_{n g}$ of $3.83,4.49$, and $4.96 \mathrm{eV} ; \mu_{n g}^{x}$ of 9.6, 4.9, and 2.3 D; and $\Delta \mu_{n g}^{x}$ of 18.7, 4.2, and 33.5 D. The state I contribution to $\beta_{x x x}^{n n}$ is largest $(90 \%)$ because both values of $\mu_{1 g}^{x}$ and $\Delta \mu_{\mathrm{I}}^{x}$ are relatively large and the excitation energy $\omega_{\mathrm{I} g}$ is lowest.

The off-diagonal component $\beta_{x x x}^{n n^{\prime}}$ is given by the expression

$$
\begin{aligned}
\beta_{x x x}^{n n^{\prime}}= & -\frac{1}{2 \hbar^{2}} \sum_{\substack{n \neq n^{\prime} \\
n \neq g \\
n^{\prime} \neq g}}\left(\mu_{g n^{\prime}}^{x} \mu_{n n^{\prime}}^{x} \mu_{n g}^{x}\right) \\
& \times\left[\left(\omega_{n g}-\omega\right)^{-1}\left(\omega_{n^{\prime} g}-2 \omega\right)^{-1}\right. \\
& +\left(\omega_{n g}-\omega\right)^{-1}\left(\omega_{n^{\prime} g}+\omega\right)^{-1} \\
& \left.+\left(\omega_{n g}+\omega\right)^{-1}\left(\omega_{n^{\prime} g}+2 \omega\right)^{-1}\right]
\end{aligned}
$$

where $\mu_{n n^{\prime}}^{x}=-\mathrm{e} r_{n n^{\prime}}^{x}$ is the $x$-component of the transition moment between excited states $n$ and $n^{\prime}$. As in the diagonal term, the important NMDVDA singlet excited states are I, III and IV with calculated gas phase values of $\mu_{\mathrm{I}, \mathrm{III}}$ : $8.3 \mathrm{D} ; \mu_{\mathrm{I}, \mathrm{IV}}: 12.8 \mathrm{D}$; and $\mu_{\mathrm{III}, \mathrm{IV}}: 4.0 \mathrm{D}$. The largest contribution to $\beta_{x x x}^{n n^{\prime}}$ results from excitations between states I and III $(59 \%)$ and states I and IV (36\%).

Density matrices of the state functions provide a compact graphical representation of important microscopic features for second order nonlinear optical processes. The transition moment $\mu_{n n^{\prime}}^{i}$ is expressed in terms of the transition density matrix $\rho_{n n^{\prime}}\left(r, r^{\prime}\right)$ by

$$
\langle\mu\rangle_{n n^{\prime}}=-\mathrm{e} \int_{r=r^{\prime}} r \rho_{n n^{\prime}}\left(r, r^{\prime}\right) \mathrm{d} r
$$

and the dipole moment difference $\Delta \mu_{n}^{i}$ by the difference density function $\rho_{n}-\rho_{g}$ between the excited and ground state functions

$$
\left\langle\Delta \mu_{n}\right\rangle=-\mathrm{e} \int r\left(\rho_{n}-\rho_{g}\right) \mathrm{d} r
$$

where $\rho_{n}$ is the first order reduced density matrix.

Contour diagrams of $\rho_{n}-\rho_{g}$ and $\rho_{n n^{\prime}}$ for states I, III and IV are plotted as examples in Figure 5 where the solid and dashed lines correspond to negative and positive values, respectively. In each case, upon virtual excitation in the second order process, large asymmetric redistribution of electron density occurs along the conjugated chain, especially in the $x$ direction, resulting in large dipole moment differences $\Delta \mu_{n}^{x}$ (Figures $5 \mathrm{a}$ and $5 \mathrm{~b}$ ) and transition moments $\mu_{n n^{\prime}}^{x}$ (Figure 5c) that determine $\beta_{x}$. Thus, for example, $\rho_{\mathrm{IV}}-\rho_{g}$ of Figure $5 \mathrm{~b}$ corresponding to $\Delta \mu_{\mathrm{IV}}^{x}$ of $33.5 \mathrm{D}$ exhibits a large correlation distance between positive and negative regions along the $x$-direction in comparison to $\rho_{\mathrm{I}}-\rho_{g}$ with its associated value of $\Delta \mu_{\mathrm{I}}^{x}$ of $18.7 \mathrm{D}$. The same charge correlated features are exhibited by $\rho_{\mathrm{I}, \mathrm{IV}}$ where the $\mu_{\mathrm{I}, \mathrm{IV}}^{x}$ of $12.8 \mathrm{D}$ is relatively large. Similar features were found in resonant benzene ring structures such as MNA. ${ }^{2,3,13,15,23}$

In summary, the understanding of the nonlinear optical responses of organic and polymeric media is reduced to experimental and theoretical studies of corresponding microscopic susceptibilities of single molecular, or polymer chain, units and the specific orientational distribution functions for the condensed assembly of such units. Two physical studies were reviewed as examples where in the first, experimental studies of third harmonic generation in a nematic liquid crystal phase demonstrate the simultaneous determination of the order parameters $\left\langle P_{2}\right\rangle$ and $\left\langle P_{4}\right\rangle$ for the general uniaxial case that also includes nematic liquid crystal polymers and uniaxially oriented polymers. In the second example, experimental and theoretical results for the frequency dependent second order nonlinear optical response $\beta_{i j k}(-2 \omega ; \omega, \omega)$ of all transpolyenyne NMDVDA show that the micro- 

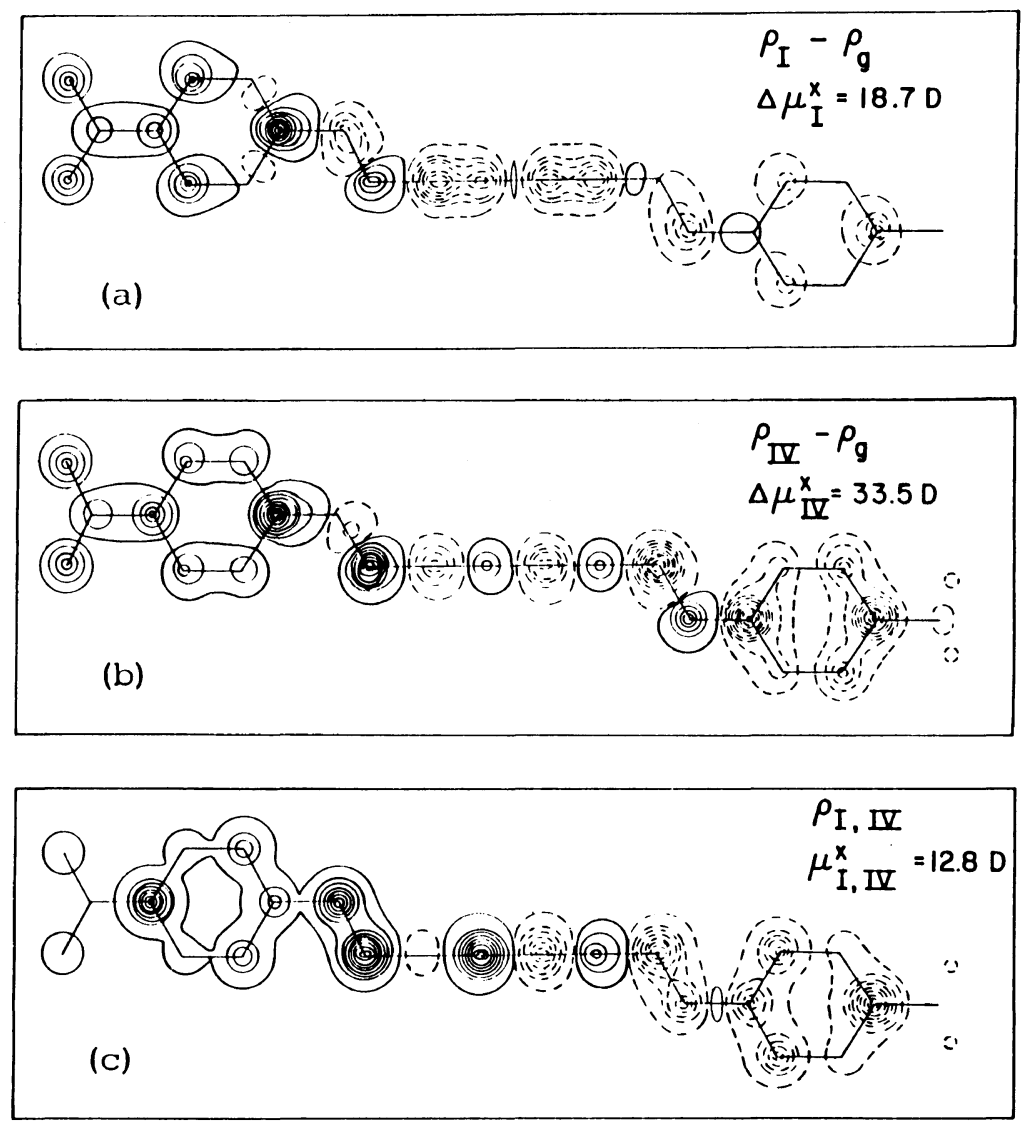

Figure 5. Contour diagrams of difference density functions for $\rho_{g}-\rho_{\mathrm{I}}(5 \mathrm{a})$ and $\rho_{g}-\rho_{\mathrm{IV}}(5 \mathrm{~b})$ and of transition density matrix $\rho_{\mathrm{I}, \mathrm{IV}}(5 \mathrm{c})$ (ref 16$)$.

scopic origin in conjugated linear chain structures resides in highly charge correlated virtual excitations of the $\pi$-electron singlet states. Such studies serve as a fundamental basis for further developing our understanding of nonlinear optical processes in organic and polymer systems.

Acknowledgements. It is a pleasure to recognize the contributions of Drs. H. T. Man, O. Zamani-Khamiri, and C. C. Teng, and Mr. Y. M. Cai. This research was supported by AFOSR and DARPA, F49620-85-C-0105 and NSF/MRL, DMR-85-19059.

\section{REFERENCES}

1. A. F. Garito and K. D. Singer, Laser Focus, 18, 59 (1982).

2. "Nonlinear Optical Properties of Organic and Polymeric Materials," D. Williams, Ed., ACS Symp. Series, Plenum Press, New York, N. Y., 1983.

3. A. F. Garito, C. C. Teng, K. Y. Wong, and O. Zammani-Khamiri, Mol. Cryst. Liq. Cryst., 106, 219 (1984).

4. Y. R. Shen, Rev. Mod. Phys., 48, 1 (1976).

5. I. R. Girling, N. A. Cade, P. V. Kolinsky, and C. M. Montgomery, Electron. Lett., 21, 169 (1985).

6. S. J. Lalama, J. E. Sohn, and K. D. Singer, SPIE Integrated Optical Circuit Engineering II, 578, 168 (1985).

7. A. F. Garito, SPIE Adv. In Mats. For Active Optics, 567, 51 (1985).

8. D. J. Williams, Angew. Chem. Intl. Ed. Eng., 23, 690 (1984). 


\section{A. F. Garito and K. Y. WoNG}

9. J. Zyss, J. Mol. Electron., 1, 25 (1985).

10. A. F. Garito, SPIE Nonlinear Optics and Applications, 613 (1986).

11. D. N. Rao, J. Swiatkiewicz, P. Chopra, S. K. Ghoshal, and P. N. Prasad, Appl. Phys. Lett., 48, 1187 (1986)

12. K. D. Singer, J. E. Sohn, and S. J. Lalama, Appl. Phys. Lett., in press.

13. S. J. Lalama and A. F. Garito, Phys. Rev., A20, 1179 (1979).

14. C. Flytzanis, "Nonlinear Optical Properties of Organic and Polymer Materials," D. Williams, Ed., ACS Symp. Ser. No. 233, Prenum Press, New York, N. Y., 1983.

15. C. C. Teng and A. F. Garito, Phys. Rev. Lett., 50, 350 (1983); Phys. Rev., B28, 6766 (1983).

16. Y. M. Cai, H. T. Man, C. C. Teng, O. ZammaniKhamiri, and A. F. Garito, XIV Int. Quant. Elec. Conf. Tech. Digest, 84 (1986); Solid State Polymerization: Structures and Properties of Polymers Produced by Lattice Controlled Processes, D. J. Sandman, Ed., ACS Symp. Series, Washington, DC, 1986, in press.

17. N. Bloembergen, "Nonlinear Optics," W. A. Benjamin, Inc., New York, N. Y., 1965.

18. K. Y. Wong and A. F. Garito, Phys. Rev., B, in press.

19. S. Jen, N. A. Clark, P. S. Pershan, and E. B. Priestly, J. Chem. Phys., 66, 4635 (1977).

20. Y. S. Lee, Y. Y. Hsu, and D. H. Dolphin,
Presentation at the ACS Symp. on Ordered Fluids and Liquid Crystals, Chicago, 1973.

21. P. I. Rose, Presentation at the IVth International Conference on Liquid Crystals, Kent, Ohio, 1972.

22. G. F. Lipscomb, R. S. Narang, and A. F. Garito, Appl. Phys. Lett., 38, 663 (1981); J. Chem. Phys., 75, 1509 (1981).

23. B. F. Levine and C. G. Bethea, J. Chem. Phys., 63, 2666 (1975).

24. D. K. Schulten, I. Ohmine, and M. Karplus, J. Chem. Phys., 64, 4222 (1976).

25. B. S. Hudson and B. E. Kohler, Chem. Phys. Lett., 14, 299 (1972); J. Chem. Phys., 59, 4984 (1973).

26. K. Y. Wong, C. C. Teng, and A. F. Garito, J. Opt. Soc. Am., B. Opt. Phys., 1, 434 (1984).

27. A. F. Garito, K. D. Singer, K. Hayes, G. F. Lipscomb, S. J. Lalama, and K. Desai, J. Opt. Soc. Am., 32, 1306 (1981).

28. J. L. Ouder, J. Chem. Phys., 67, 446 (1977).

29. K. D. Singer and A. F. Garito, J. Chem. Phys., 75, 3572 (1981).

30. $\mathrm{CNDO} / \mathrm{s}$ parameters optimized for optical excitations with standard bond lengths and angles for all trans geometry. Calculated components of $\mu: \mu_{x}=$ 9.4, $\mu_{y}=-0.5$ and $\mu_{z} \simeq 0 \mathrm{D}$ with $\mu$ at an angle of $3^{\circ} \simeq 0^{\circ}$ to $x$-axis, in which case $\mu=\mu_{x} \cos \theta+$ $\mu_{y} \sin \theta \simeq \mu_{x}$ and $\beta_{x}=\beta_{x x x x}+1 / 3\left(\beta_{x y y}+\beta_{x x z}+2 \beta_{y y z}\right.$ $+2 \beta_{z z x}$ ). 